\title{
Doctors, pharmacists push back on medical abortion rules
}

Cite as: CMAJ 2017 March 27;189:E480-1. doi: 10.1503/cmaj.1095406

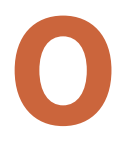

ntario doctors and pharmacists have the greenlight from their regulatory colleges to ignore some of Health Canada's rules for dispensing the abortion medication Mifegymiso, a two-drug combination of mifepristone, also called RU-486, and misoprostol.

Health Canada approved Mifegymiso in 2015, but the drug is almost impossible to access outside of a handful of clinics in major cities. Doctors and pharmacists say the biggest barrier is that Health Canada only allows specially trained physicians to dispense the drug. This means a doctor has to stock Mifegymiso or send patients to a pharmacist who can courier it back to the doctor for hand off. Health Canada also requires that the physician "supervise" administration of the drug, but leaves it to the prescriber to decide if a patient has to ingest it in their presence.

These rules are not "legally binding," according to new guidance from the College of Physicians and Surgeons of Ontario and the Ontario College of Pharmacists. They argue "it is within the scope of practice of pharmacists in Ontario to dispense medications directly to patients," and that includes Mifegymiso. From Health Canada's standpoint, this is an "off-label" use of the drug. Both physicians and pharmacists would still be required to undergo the mandatory training offered by the Society of Obstetricians and Gynaceologists of Canada (SOGC).

The Ontario guidance echoes advice issued by the colleges' counterparts in British Columbia in January. The new guidance "doesn't completely absolve pharmacists of any potential liability," says Phil Emberley, director of professional affairs at the Canadian Pharmacists Association. "But a lot of pharmacists will see this as an opportunity to serve Canadians better, so I don't see this being a huge problem."

Ontario's stance will likely set a precedent for other provincial colleges to follow suit, Emberley adds. Ideally, Health Canada would change its rules so that doctors and pharmacists aren't forced to practise off-label. Until then, many may simply err on the side of caution and choose not to participate at all, as already seems to be the case. Even requiring doctors and pharmacists to undergo extra training to provide the drug "limits the pool," Emberley says.

Health Canada claims it has imposed these hurdles "to help reduce risks to patients." But Emberley and others argue there's no scientific basis for imposing greater restrictions on Mifegymiso over other equally or more dangerous drugs that are prescribed and dispensed normally.

Mifegymiso has been the gold standard for medical abortion in many other developed countries for decades. "Clinicians should be reassured that the medication is very safe, and in other jurisdictions, it's quite clear that private provision and self-administration is safe," says Dr. Dustin Costescu, a family planning specialist at McMaster University in Hamilton, Ontario, who wrote the SOGC training program for Mifegymiso. "I'm unaware of any reports of abuse, so the

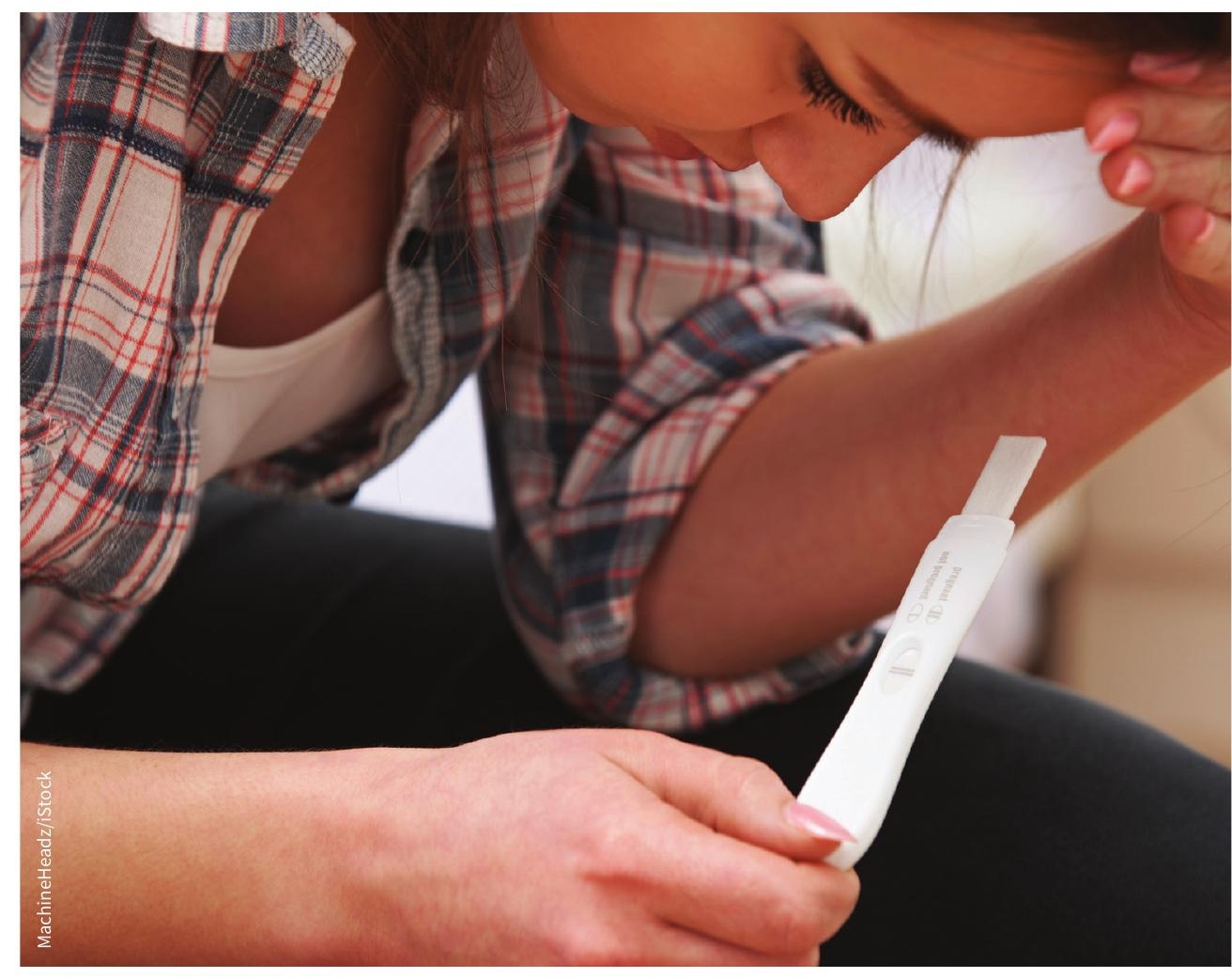

Colleges of physicians and pharmacists suggest off-label workaround for abortion drug restrictions. 
fear of somebody else getting the medication is unfounded."

Dr. Wendy Norman, a family planning expert at the University of British Columbia, argues the restrictions are more politically motivated than scientifically grounded. "This needs to be taken out of that advocacy range; this is a health issue," she said. "We all know that women can have extraordinary and rare ill effects from birth control pills, from pregnancy ... these are as or more dangerous than dealing with Mifegymiso."

Confusion about the pharmacist's role may also be contributing to supply problems. Some doctors are holding off on prescribing the drug until it's available in local pharmacies, but pharmacists are unaware "the only way to get the medicine is to have completed the training program," says Norman. "So there may be pharmacists all around the country who are waiting until their distributor lets them know they've got it, when in fact that's not the first step at all; they have to go to the SOGC's website."

Recently, the manufacturer Celopharma also began asking physicians who completed the training to get their patients to bring a signed consent form with them to the pharmacist, to prove that their doctor was licensed to prescribe Mifegymiso. "This causes huge confidentiality issues because the woman can be visually and physically identified as somebody having an abortion," Norman says.

Originally, this confirmation was going to be handled through a centralized registration system, says Costescu, but "Health Canada felt that process was not rigorous enough."

In small communities, this has the potential to expose the doctor, pharmacist and patient to backlash from people who oppose abortion, he said. "In a system where there are very few providers, that's actually where safety becomes an issue."

The seven-week window (from the first day of the last menstrual period) that women have to access Mifegymiso and the high cost of the drug (about $\$ 300$ ) are further barriers to access. For women living in rural Canada, who already have to wait longer and travel farther for abortion services, the window is particularly tight, says Emberley. It may take weeks for a woman to learn they are pregnant, let alone to book multiple appointments with a doctor who prescribes the drug.

When it comes to the cost of Mifegymiso, Costescu says many physicians have told him "they don't see patients being able to afford the drug, so they're reluctant to stock it in the first place." The manufacturer has applied to Health Canada to expand the window of eligibility for Mifegymiso and allow pharmacist dispensing, and the Canadian Agency for Drugs and Technologies in Health (CADTH) Common Drug Review is studying whether public drug plans should cover the prescription.

Norman does not buy that Health Canada must wait on drug companies to update its recommendations. "Health Canada needs to have a process whereby it can make those revisions without requiring the company to take the initiative. This is an initiative that belongs with our government."

Lauren Vogel, CMAJ 